\title{
Evaluation of frailty status and prognosis in patients aged over 75 years with chronic kidney disease (CKD)
}

\author{
Masaki Yoshida*, Yurie Takanashi, Takako Harigai, Noriyuki Sakurai, Keiko Kobatake, Hiroaki Yoshida, \\ Satsuki Kobayashi, Takayuki Matsumoto and Kazue Ueki
}

\begin{abstract}
Background: There is a higher frequency of advanced chronic kidney disease (CKD) in frail patients than in the general population. This study evaluated frailty status before initiation of dialysis and clarified the prognosis in patients aged over 75 years with advanced CKD.

Method: This study involved 310 patients who initiated dialysis between January 2011 and December 2018. Frailty was evaluated using the Rockwood Clinical Frailty Scale (CFS). Age, sex, body mass index (BMI), laboratory data, the Charlson Comorbidity Index (CCl), geriatric syndrome (based on SPICES score), nutritional status (based on the Controlling Nutritional Status [CONUT] score), and the effects of frail conditions on the prognosis were examined.

Results: There were 107 robust participants (34.5\%), 100 pre-frail participants (32.3\%), and 103 frail participants (33.2\%). The median survival time was significantly different among the robust (54.3 months), pre-frail (39.7 months), and frail participants (18.7 months) by the log-rank test $(P<0.001)$. HR of frail group compared to robust group was $1.59(P=0.04)$. Pre-frail group did not show a significantly higher hazard than frail group. The other significant variables maintained in the model were CONUT score $(P<0.001), C C l$, and SPICES score. The Kruskal-Wallis test showed that CONUT score $(P<0.001)$, SPICES score $(P<0.001)$, and $C C I(P=0.013)$ were significant differences in three independent groups (robust, pre-frail, frail).
\end{abstract}

Conclusion: Frail patients receiving dialysis have a poor prognosis. Frailty was associated with comorbidities, nutrition, and especially geriatric syndrome.

Keywords: Frail, Clinical Frailty Scale, Charlson Comorbidity Index, SPICES score, CONUT score

\section{Introduction}

Frailty is a major public health problem in the older population. It has been recently defined by the International Association of Gerontology and Geriatrics Frailty Consensus as reduced strength and physiologic malfunctioning that increases an individual's susceptibility to increased dependency, vulnerability, and death [1]. Frailty can be used as a marker of adverse outcome risk

\footnotetext{
* Correspondence: masakiyoshida-knz@umin.ac.jp Dialysis and Nephrology Center, Sanshikai TOHO Hospital, 1155 Azami, Kasagake-machi, Midori, Gunma 379-2311, Japan
}

in older adults and is increasingly used to predict patient outcomes across specialties, such as nephrology, oncology, cardiology, and orthopedics.

A systematic review and meta-analysis identified five studies incorporating 11,940 Japanese people aged 65 years or older living in the community and demonstrated that the pooled prevalence of frailty, pre-frailty, and robustness based on the Fried criteria were 7.4, 48.1, and $44.4 \%$, respectively. Stratified analyses showed that women were frailer than men and that the prevalence of frailty increased with age [2].

(c) The Author(s). 2020 Open Access This article is licensed under a Creative Commons Attribution 4.0 International License, which permits use, sharing, adaptation, distribution and reproduction in any medium or format, as long as you give appropriate credit to the original author(s) and the source, provide a link to the Creative Commons licence, and indicate if changes were made. The images or other third party material in this article are included in the article's Creative Commons licence, unless indicated otherwise in a credit line to the material. If material is not included in the article's Creative Commons licence and your intended use is not permitted by statutory regulation or exceeds the permitted use, you will need to obtain permission directly from the copyright holder. To view a copy of this licence, visit http://creativecommons.org/licenses/by/4.0/ The Creative Commons Public Domain Dedication waiver (http://creativecommons.org/publicdomain/zero/1.0/) applies to the data made available in this article, unless otherwise stated in a credit line to the data. 
There are no established criteria for diagnosing frailty, but the Cardiovascular Health Study (CHS) criteria for frailty based on Fried's phenotype model (FP) [3] and the frailty index (FI) established by Mitnitski et al. and defined as a proportion of accumulated deficits [4] are the main methods. More recently, in the Asia-Pacific region, CFS has emerged as a well-validated 9-point global assessment tool that predicts adverse outcomes in older adults. The CFS allows frailty to be defined and graded using simple clinical descriptors available from routine clinical assessment [5-8].

The reference standard for diagnosing frailty in CKD patients, FP, is a time-consuming evaluation and therefore challenging to use outwith the research environment.

The CFS was the most effective screening method of frailty, comparable to that of the FP, suggesting it is a useful test offering prognostic value. Considering that the CFS has also been demonstrated to be an accurate screening tool for frailty, as defined by the FP [9], Nixon et al. recommend its use in patients with advanced CKD and encourage systematic frailty screening programs within nephrology services.

In a report examining the relationship between frailty and stage of chronic kidney disease (CKD) in predialysis patients in a hospital in Korea, the frequency of frailty increased as the stage of CKD progressed (stages $1-2$, 5.9-14.0\%; stages 3-5, 10.7-56.0\%). The frail patients had a notably reduced physical and mental quality of life, as measured by the Short Form-36 Health Survey, indicating that frailty is a risk factor for mortality or renal replacement therapy (odds ratio [OR] 2.0-2.5) [10-12]. The frequency of frailty in dialysis patients is 13.8-66.7\%, which is higher than in the general population and conservative CKD patients, and frailty is of prognostic value for dialysis patients [13].

There is an increasing trend of chronic dialysis patients in Japan. The number reached about 330,000 at the end of 2017. The average age of these patients was 68.43 years, and $34.2 \%$ were aged 75 years and over. The number of newly initiated dialysis patients is also increasing every year. In 2017, the average age of these patients was 69.68 years, and $41.6 \%$ were aged 75 years and over [14].

In this study, we evaluated the frequency of frailty and the factors associated with frailty in patients aged over 75 years with CKD (stages 4-5). The aim of this study examined the correlation among CFS and other indices representing comorbidities, nutritional disorders, and geriatric syndrome, considering the prognosis.

\section{Materials and methods Study design and sample}

We conducted a prospective cohort study of 751 patients initiated dialysis between January 1, 2011, and December 31, 2018, at TOHO Hospital, Gunma, Japan.

TOHO Hospital is a certified facility recognized by the Japanese Society for Dialysis Therapy. Patients' characteristics included age, sex, body mass index (BMI), cardiothoracic ratio (CTR), laboratory data [serum albumin (Alb), serum sodium, serum potassium, estimated glomerular filtration rate (eGFR), serum chloride, corrected serum calcium, serum phosphorus, blood urea nitrogen, hemoglobin, brain natriuretic peptide (BNP), total cholesterol, total lymphocyte count, and C-reactive protein (CRP)], the Charlson Comorbidity index (CCI) as comorbidity, SPICES score as a measure of geriatric syndrome, Controlling Nutritional Status (CONUT) score as a measure of nutrition, and Rockwood's Clinical Frailty Scale (CFS) as a measure of frailty. Baseline clinical and laboratory data were collected from an electronic medical chart before dialysis was initiated. Comorbidity data were obtained from clinic letters of each patient prior to admission. The presence or absence of each comorbid condition was verified with the patient at the time dialysis was initiated. A detailed assessment of each patient's functional abilities and level of dependency was documented in the admission report by nurses and doctors. The report is written according to a pre-specified template. The template includes sections on comorbidity, patient mobility, ability to cope with activities of daily living, and the level of social support available or required. The reports contain all the information required to generate a CFS, CCI, and SPICES score for each patient.

Inclusion criteria were patients aged over 75 years. Exclusion criteria were patients on peritoneal dialysis; patients whom doctors found difficult to assess in terms of medical history, clinical course, and daily life before admission; and patients with a CFS score of 9 .

In total, 359 patients were considered eligible for this study. However, 49 patients who had undergone maintenance dialysis outside TOHO Hospital and three related facilities (Oura Hospital, Hikari Clinic, Shirota Clinic) after the initiation of dialysis were excluded, resulting in a study sample of 310 patients (Fig. 1).

\section{Frailty screening in the clinical setting: Rockwood's CFS}

More recently, in the Asia-Pacific region, the Clinical Frailty Scale (CFS) has emerged as a well-validated 9point global assessment tool that predicts adverse outcomes in older adults. The CFS allows frailty to be defined and graded using simple clinical descriptors available from routine clinical assessment. However, the tool requires some clinical judgment, and trained assessors are required for accurate classification [5-8]. 


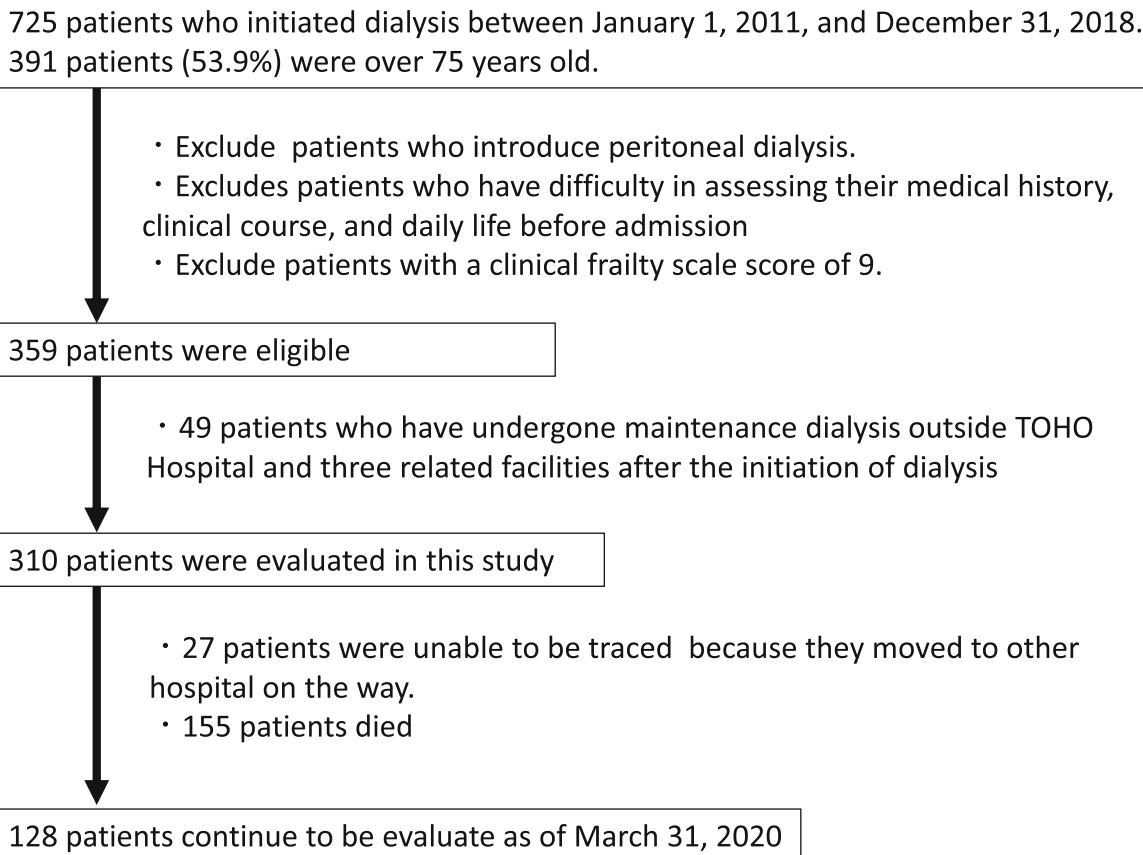

Fig. 1 Study design

1. Very fit

People who are robust, active, energetic, and motivated. These people commonly exercise regularly. They are among the fittest for their age.

2. Well

People who have no active disease symptoms but are less fit than category. Often, they exercise or are very active occasionally, e.g., seasonally.

3. Managing well

People whose medical problems are well controlled but are not regularly active beyond routine walking.

4. Vulnerable

While not dependent on others for daily help, often symptoms limit activities. A common complaint is being "slowed up" and/or being tired during the day.

5. Mildly frail

These people often have more evident slowing and need help in high order instrumental activities of daily living (IADLs) (finances, transportation, heavy housework, medications). Typically, mild frailty progressively impairs shopping and walking outside alone, meal preparation, and housework.

6. Moderately frail

People need help with all outside activities and with keeping house. Inside, they often have problems with stairs and need help with bathing and might need minimal assistance (cuing, standby) with dressing.
7. Severely frail

Completely dependent for personal care, from whatever cause (physical or cognitive). Even so, they seem stable and not at high risk of dying (within-6 months).

8. Very severely frail Completely dependent, approaching the end of life. Typically, they could not recover even from a minor illness.

9. Terminally ill Approaching the end of life. This category applies to people with a life expectancy $<6$ months, who are not otherwise evidently frail.

In this study, terminally ill was excluded.

According to Rockwood's CFS, the patients were divided into three groups: robust (CFS 1-3), pre-frail (CFS 4), and frail (CFS 5-8) [5].

\section{Charlson comorbidity index}

This is a health tool based on the CCI model that assesses the comorbidity risk associated to a series of conditions in order to offer medical specialists an informed decision-making process in terms of specific screenings or medical procedures. The index accounts for the patient age and 16 conditions. This instrument is used to categorize comorbidities of patients and uses the International Classification of Diseases (ICD) diagnosis codes [15]. 


\section{SPICES score}

SPICES is an acronym for a brief protocol for multidimensional assessment to identify risk factors related to caring for older adults: skin integrity, problem with eating, incontinence, confusion, evidence of falls, and sleep disturbance.

Skin integrity is documented presence of a pressure ulcer on admission by a registered nurse and/or physician. Problem with eating are evaluated by Functional Independence Measure (FIM) [16]. Incontinence (bowel and/or bladder) is evaluated by FIM. Confusion is evaluated by screening at admission (Delirium Screening Tool). Evidence of falls is defined as fracture history of falls within past year.

Sleep disturbance is defined as insomnia, restless leg syndrome, periodic limb movements in sleep, and sleep apnea syndrome. All factors are scored as one, and the total score is evaluated [17].

\section{Controlling Nutritional Status score}

The CONUT considers the Alb level, total cholesterol level, and total lymphocyte count. Alb scores were 0 ( $\geq$ $3.5 \mathrm{~g} / \mathrm{dL}), 2(3.0-3.4 \mathrm{~g} / \mathrm{dL}), 4(2.5-2.9 \mathrm{~g} / \mathrm{dL})$, and 6 points $(<2.5 \mathrm{~g} / \mathrm{dL})$. Total cholesterol score was $0(\geq 180 \mathrm{mg} /$ $\mathrm{dL}), \quad 1(140-179 \mathrm{mg} / \mathrm{dL}), 2(100-139 \mathrm{mg} / \mathrm{dL})$, and 3 points $(<100 \mathrm{mg} / \mathrm{dL})$. Total lymphocyte score was $0(\geq$ $1600 / \mu \mathrm{L}), 1(1200-1599 / \mu \mathrm{L}), 2(800-1199 / \mu \mathrm{L})$, and 3 points $(<800 / \mu \mathrm{L})$. Each score was totaled and evaluated. Patients with a total score of $\geq 2$ were considered to have malnutrition [18].

\section{Statistical analysis}

The Kruskal-Wallis test and Pearson's $\chi^{2}$ test were performed for comparing three independent groups (robust, pre-frail, frail). As a method of multiple comparisons between groups, the Steel-Dwass test and the MannWhitney $U$ test were performed for two-group comparisons, and Bonferroni adjustments were conducted where appropriate.

Survival times after dialysis initiation of patients under different frailty conditions were evaluated by a longitudinal cohort study. The Kaplan-Meier method was used to examine crude survival in the three groups defined by their frailty status (frail, pre-frail, and robust). Cox proportional hazards regression was applied firstly to estimate unadjusted hazard ratios (HRs) in the three groups. Next, multidimensional Cox proportional hazards regression model was used to adjust for possible confounders. This was performed by entering all the variables potentially associated with survival into the model: Rockwood's CFS, CCI, SPICES score, CONUT score, age, sex, BMI, CTR, laboratory data (Alb, serum sodium, serum potassium, serum creatinine, serum chloride, corrected serum calcium, serum phosphorus, blood urea nitrogen, hemoglobin, BNP, total cholesterol, total lymphocyte count, and (RP). The Cox proportional hazards regression modeling results were summarized with HRs for each variable, 95\% confidence intervals, and associated $p$ values.

Statistical analysis was conducted using SPSS24 $4^{\circ}$ for Windows.

\section{Ethics}

This study was performed with the approval of the Institutional Review Board of our institution.

\section{Results}

The median follow-up period after dialysis initiation was 27.3 months (interquartile range [IQR] 8.0-46.2), and the average was 29.2 months (standard deviation $[\mathrm{SD}] \pm$ 24.2).

Patient data are shown in Table 1 . The median age was 83.1 years (interquartile range [IQR] 80.6-86.7), and 144 were women $(45.4 \%)$. The median eGFR at the time of evaluation was $8.5 \mathrm{ml} / \mathrm{min} / 1.73 \mathrm{~m}^{2}$ (IQR 5.6-10.9). The median CCI was 4 (IQR 3-5), the median CONUT score was 6 (IQR 4-8), and the median SPICES score was 1 (IQR $0-2$ ). The primary causes of CKD were diabetes mellitus $(n=95,30.5 \%)$, glomerulonephritis $(n=48,15.4 \%)$, nephrosclerosis $(n=35,11.3 \%)$, and autosomal-dominant polycystic kidney disease $(n=7,2.3 \%)$.

\section{Frailty}

The status of frailty before initiation of dialysis was classified by Rockwood's CFS. Patients were divided into three groups: robust $(n=107$; CFS $=1-3,34.5 \%)$, prefrail $(n=100$; CFS $=4,32.3 \%)$, and frail $(n=103$; CFS $=$ 5-8, 33.2\%) (Fig. 2).

\section{Prognosis after dialysis initiation}

As of March 31, 2020, 27 patients (8.7\%) were censored because they were transferred to another hospital during maintenance dialysis, and 155 patients (50.0\%) died.

The causes of death and the number of patients are shown in Fig. 3. Infection was the leading cause of death $(16.8 \%)$. In the frail group, mortality of infectious diseases tended to be high, and in the robust group, mortality of heart disease tended to be high.

For the prognosis after initiation of dialysis, the Kaplan-Meier curve was constructed. The median survival was 54.3 months (95\% confidence interval [CI], $36.6-71.9$ ) in the robust group, 39.7 months (95\% CI, $21.7-57.8$ ) in the pre-frail group, and 18.7 months (95\% $\mathrm{CI}, 5.3-32.2)$ in the frail group, and a significant difference was found by log-rank analysis $(P<0.001)$.

Cumulative survival rates at 12,36 , and 60 months after initiation of dialysis were $85.7,72.5$, and $45.8 \%$ 
Table 1 Characteristics of all study participants with frailty classification and frailty status. Values of categorical variables are given as number (percentage). Values for continuous variables are given as median (interquartile range)

\begin{tabular}{|c|c|c|c|c|c|}
\hline Characteristic & Total $(N=310)$ & Robust $(N=107)$ & Pre-Frail $(N=100)$ & Frail $(N=103)$ & $P$-value \\
\hline Age (year) & $83.1(80.6-86.7)$ & $82.6(80.6-85.3)$ & $82.9(80.4-87.1)$ & $83.8(81.2-87.7)$ & 0.124 \\
\hline Sex (Female) & $144(45.4)$ & $38(32.3)$ & $55(55.1)$ & $51(49.0)$ & $0.027^{*}$ \\
\hline Body mass index (kg/m2) & $19.7(17.5-22.0)$ & $19.6(18.2-22.0)$ & $19.6(17.4-21.9)$ & $18.5(15.9-21.6)$ & $0.028^{*}$ \\
\hline Cardio-Thoracic Ratio (\%) & $54.5(50.0-60.8)$ & $55(50-61)$ & $54.4(51-60)$ & $55(5061)$ & 0.828 \\
\hline Charlson Comorbidity index & $4(3-5)$ & $4.0(3.0-5.0)$ & $4.0(3.0-5.0)$ & $4.0(3.0-6.0)$ & $0.013^{*}$ \\
\hline Cardiovascular disease & $57(18.4)$ & $18(16.8)$ & $23(23.0)$ & $16(15.5)$ & 0.388 \\
\hline Congestive heart failure & $80(25.8)$ & $28(26.2)$ & $26(26.0)$ & $26(25.2)$ & 0.924 \\
\hline Peripheral vascular disease & $12(3.9)$ & $5(4.7)$ & $4(4.0)$ & $3(2.9)$ & 0.791 \\
\hline Dementia & $89(28.7)$ & $12(11.2)$ & $26(26.0)$ & $51(49.5)$ & $<0.001^{* *}$ \\
\hline Chronic obstructive pulmonary disease & $15(4.8)$ & $4(3.7)$ & $4(4.0)$ & $7(6.8)$ & 0.519 \\
\hline Collagen disease & $16(5.2)$ & $7(6.5)$ & $5(5.0)$ & $4(3.9)$ & 0.680 \\
\hline Peptic ucler & $5(1.6)$ & $1(0.9)$ & $1(1.0)$ & $3(2.9)$ & 0.436 \\
\hline Mild Liver dysfunction & $10(3.2)$ & $4(3.7)$ & $3(3.0)$ & $3(2.9)$ & 0.917 \\
\hline Diabetes mellitus without end-organ damage & $22(7.1)$ & $2(1.9)$ & $11(11.0)$ & $9(8.7)$ & 0.599 \\
\hline Hemiplegia & $46(14.8)$ & $11(10.3)$ & $13(13.0)$ & $22(21.4)$ & 0.149 \\
\hline Diabetes mellitus with end-organ damage & $107(34.5)$ & $40(37.4)$ & $35(35.0)$ & $32(31.1)$ & 0.650 \\
\hline Malignant Tumor (no metastasis) & $22(7.1)$ & $6(5.6)$ & $8(8.0)$ & $8(7.8)$ & 0.765 \\
\hline Moderate/Sever Liver dysfunction & $6(1.9)$ & $0(0.0)$ & $4(4.0)$ & $2(1.9)$ & 0.122 \\
\hline Metastatic malignant tumor & $4(1.3)$ & $0(0.0)$ & $1(1.0)$ & $3(2.9)$ & 0.178 \\
\hline SPICES Score & $0(0-3.0)$ & $0.0(0.0-1.0)$ & $1.0(0.0-1.5)$ & $2.0(1.0-3.0)$ & $<0.001^{* *}$ \\
\hline Sleep disorder & $67(21.6)$ & $30(28.0)$ & $18(18.0)$ & 19 (18.4) & 0.13 \\
\hline Problem with eating & $92(29.7)$ & $9(8.4)$ & $26(26.0)$ & $57(55.3)$ & $<0.001^{* *}$ \\
\hline Incontinence & $93(30.0)$ & $8(7.5)$ & $22(22.0)$ & $63(61.2)$ & $<0.001^{* *}$ \\
\hline Confusion & $89(28.7)$ & $12(11.2)$ & $26(26.0)$ & $51(49.5)$ & $<0.001^{* *}$ \\
\hline Evidence of Falls & $34(11.0)$ & $6(5.6)$ & $10(10.0)$ & $18(17.5)$ & $0.021^{*}$ \\
\hline Skin breakdown & $18(5.8)$ & $1(0.9)$ & $2(2.0)$ & $15(14.6)$ & $<0.001^{* *}$ \\
\hline \multicolumn{6}{|l|}{ Etiology of chronic kidney disease } \\
\hline Diabetes mellitus & $101(32.6)$ & $37(34.6)$ & $33(33.0)$ & $31(30.1)$ & 0.452 \\
\hline Glomeruonephritis & 49 (15.8) & $16(15.0)$ & $16(16.0)$ & $17(16.5)$ & 0.591 \\
\hline Nephrosclerosis & $38(12.3)$ & $13(12.1)$ & $13(13.0)$ & $12(11.7)$ & 0.206 \\
\hline Other & $16(5.2)$ & $6(5.6)$ & $6(6.0)$ & $4(3.9)$ & 0.353 \\
\hline Unknown & $107(34.5)$ & $35(32.7)$ & $33(33.0)$ & 39 (37.9) & 0.225 \\
\hline \multicolumn{6}{|l|}{ Labodata } \\
\hline Serum Albumin (g/dl) & $2.9(2.2-3.4)$ & $3.1(2.8-3.4)$ & $2.9(2.4-3.3)$ & $2.6(2.2-3.0)$ & $<0.001^{* *}$ \\
\hline Hemoglobin (g/dl) & $9.0(7.8-10.1)$ & $8.8(8.3-10.1)$ & $8.9(8.0-9.9)$ & $8.8(7.8-9.9)$ & 0.452 \\
\hline Serum Sodium (mEq/l) & $137.6(133-141)$ & $138(136-141)$ & $138(135-141)$ & $138(133-141)$ & 0.591 \\
\hline Serum Potassium (mEq/l) & $4.5(3.9-5.4)$ & $4.3(3.9-5.0)$ & $4.3(3.8-4.9)$ & $4.6(3.9-5.4)$ & 0.206 \\
\hline Serum Chloride (mEq/l) & $105.8(110-111)$ & $107(103-111)$ & $106(101-110)$ & $106(101-110)$ & 0.24 \\
\hline Blood Urea Nitrogen (mg/dl) & $81.4(54.0-111)$ & $72.5(57.4-88.6)$ & $73.2(54.0-93.6)$ & $75.3(60.8-111)$ & 0.156 \\
\hline Serum Creatinine (mg/dl) & $5.3(4.0-6.6)$ & $5.6(4.2-7.2)$ & $4.9(4.0-6.2)$ & $5.2(4.3-7.4)$ & 0.078 \\
\hline Corrected Serum Calcium (mg/dl) & $9.3(8.9-9.9)$ & $9.3(8.8-9.5)$ & $9.3(8.9-9.6)$ & $9.6(9.2-9.9)$ & $<0.001^{* *}$ \\
\hline Serum Phosphorus (mg/dl) & $5.1(3.8-6.2)$ & $4.7(3.9-5.5)$ & $4.6(3.8-5.5)$ & $5.2(4.2-6.2)$ & $0.012^{*}$ \\
\hline Brain Natriuretic Peptide (pg/ml) & $196(72.2-570)$ & $162.5(76-405)$ & $147(63-458)$ & $162.5(76-405)$ & 0.432 \\
\hline
\end{tabular}


Table 1 Characteristics of all study participants with frailty classification and frailty status. Values of categorical variables are given as number (percentage). Values for continuous variables are given as median (interquartile range) (Continued)

\begin{tabular}{llllll}
\hline Characteristic & Total $(\boldsymbol{N}=\mathbf{3 1 0})$ & Robust $(\boldsymbol{N}=\mathbf{1 0 7})$ & Pre-Frail $(\boldsymbol{N}=\mathbf{1 0 0})$ & Frail $(\boldsymbol{N}=103)$ & $P$-value \\
\hline Total Cholesterol $(\mathrm{mg} / \mathrm{dl})$ & $161(136-192)$ & $167(141-197)$ & $159(130-180)$ & $160(131-193)$ & 0.161 \\
Total Lymphocyte Count $(1000 / \mu \mathrm{L})$ & $0.98(0.70-1.35)$ & $0.99(0.8-1.4)$ & $1.01(0.8-1.4)$ & $0.93(0.6-1.2)$ & 0.083 \\
C-reactive protein $(\mathrm{mg} / \mathrm{dl})$ & $0.7(0.72-1.44)$ & $0.16(0.06-0.76)$ & $0.78(0.07-1.42)$ & $1.12(0.42-1.95)$ & $<0.001^{* *}$ \\
CONUT score & $6(4.0-8.0)$ & $5(4-7)$ & $6(4-8)$ & $7(6-9)$ & $<0.001^{* *}$ \\
\hline
\end{tabular}

* $P$ value $<0.05$

** $P$ value $<0.001$

(robust group); 75.3, 53.2, and 31.0\% (pre-frail group); and $52.7,39.7$, and $16.1 \%$ (frail group), respectively (Fig. 4).

In the Cox proportional hazards model of patient survival, the unadjusted hazard rate of mortality for frailty, using the robust group as a reference, was 1.64 (95\% CI, 1.07-2.53; $P=0.025$ ) and 3.13 (95\% CI, 2.084.73; $P<0.001)$ for pre-frail and frail group, respectively. When adjusted by the other significant variables for mortality, HR of frail compared to robust group was 1.59 (95\% CI, 1.10-2.58; $P<0.001)$. Pre-frail group did not show a significantly higher hazard than robust group. The other significant variables maintained in the multivariable Cox proportional hazards model were CCI, SPICES score, and CONUT score. HR of CONUT score was 1.13 (95\% CI, $1.05-1.21 ; P=0.001$ ), HR of CCI was 1.17 (95\% CI, 1.08$1.27 ; P=0.001)$, and HR of SPICES score was $1.29(95 \%$ CI, 1.14-1.47; $P=0.002$ ) (Fig. 5, Table 2).

CONUT score, SPICES score, and CCI showed significant differences by the Kruskal-Wallis test. Furthermore, in the comparison of the groups (robust, pre-frail, frail), SPICES scores were distinct between all groups; CONUT score and CCI were distinct among some groups (Fig.6a-c, Table 1).

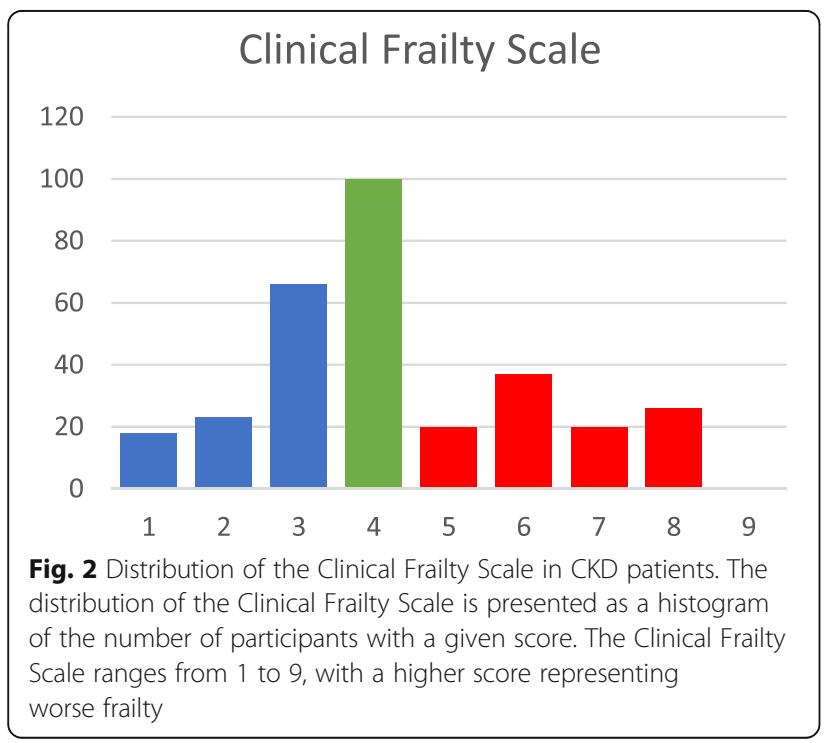

The correlation between CFS and CONUT score, SPICES score, and CCI was examined using Spearman's rank correlation coefficient. CFS was positively correlated with all indicators, with a significance probability of less than $5 \%$ (Table 3).

\section{Discussion}

It is important to evaluate frailty because it is the number one cause of nursing care needs in people aged over 75 years. Frailty is an independent predictor of adverse outcomes in chronic kidney disease. The number of Japanese CKD patients is estimated to be about 13.3 million, and about one in eight adults has CKD. CKD prevalence is particularly high in the elderly [19]. In a systematic review of cohorts and observational studies assessing the association of frailty with CKD and prognosis, CKD was associated with frailty or reduced physical function (OR 1.30-3.12). It has already been shown that frailty is associated with the initiation of dialysis or death in CKD patients (OR 2.00-5.88) [20].

As a result of examining the relationship between eGFR and frailty, the frequency of frailty increased as the eGFR decreased. The ORs for CKD stage 4 and CKD stage 5 are 2.02 and 4.83, respectively, compared to CKD stage 1-2 [21]. In a report of CFS's assessment of frailty in dialysis patients, $26 \%$ were frail, and a 1point increase in CFS increased the risk of death by 1.22 times (95\% CI 1.04-1.43) [13].

As shown in Table 1, the proportion of females was higher in the pre-frail/frail group than in the robust group. Although the pathophysiological pathways leading to frailty are not well defined, gender appears to be an important factor affecting the aging trajectory. Compared with age-matched males, females tend to be frail but have a higher life expectancy [22].

It is known that female hormone levels decrease with menopause. In comparison to males, females are more likely to develop dementia and osteoporosis, thereby affecting ADL and QOL decline. In addition, females have a relatively long life expectancy and healthy life expectancy. However, the differences between both life expectancy and the length of care required also become longer, and ADL and QOL are likely to decline. The Kaplan-Meier curve 


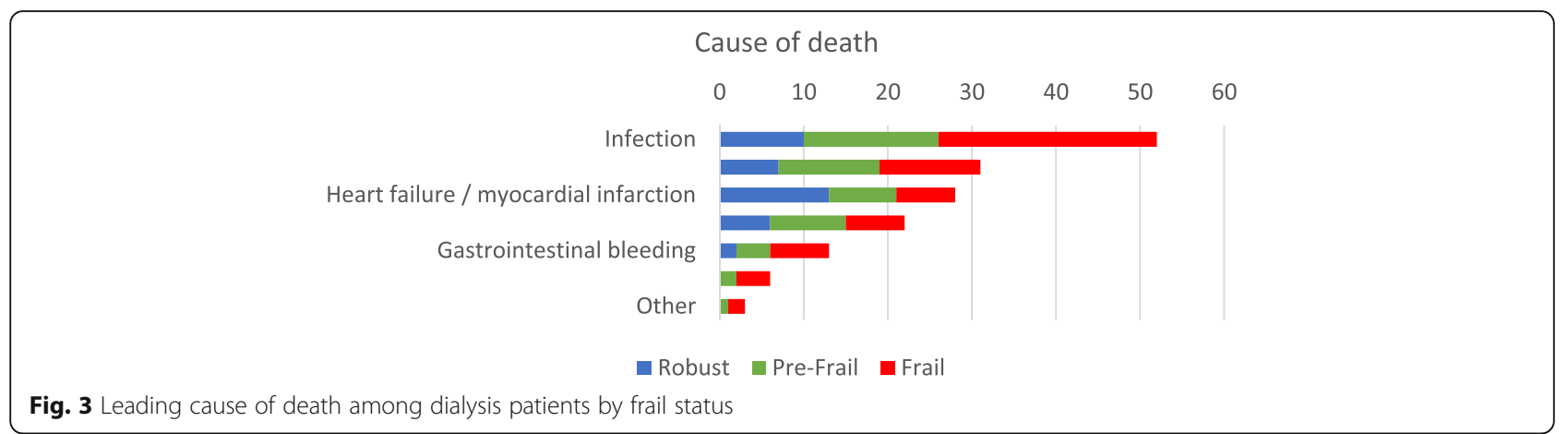

in Fig. 4 shows a sharp decrease in survival until 12 months after the introduction of dialysis. Especially in the frail group, the tendency is remarkable, and the survival rates at 3,6 , and 12 months were 76 , 62.8 , and $55.9 \%$. In previous reports, only severe (bedridden) and moderately (overt difficulties in exerting basic activities of daily living) impaired functional status was significantly associated with early mortality after initiation of dialysis (adjusted risk ratio 3.93 and 2.38, respectively) [23]. Functional status among older people with severe and moderate disabilities is consistent with the frail group classified by the CFS. Physical function in older people may be further reduced after the introduction of dialysis. In a study of nursing home residents, the proportion of deaths or reduced functional status among the residents was $61 \%$ compared with predialysis within 3 months after initiation of dialysis, and $39 \%$ had the same functional status as before dialysis. By 12 months, the proportion of deaths or reduced functional status among the residents was $87 \%[24]$.

\section{Comorbidity}

Among the groups, the frail patients had a significantly higher CCI. Of the diseases that make up CCI, only dementia showed a significant difference, with 51 (49.5\%) in the frail group. Cross-sectional studies show that about $20-55 \%$ of frail patients have cognitive impairment [25]. In a longitudinal study, the risk of having

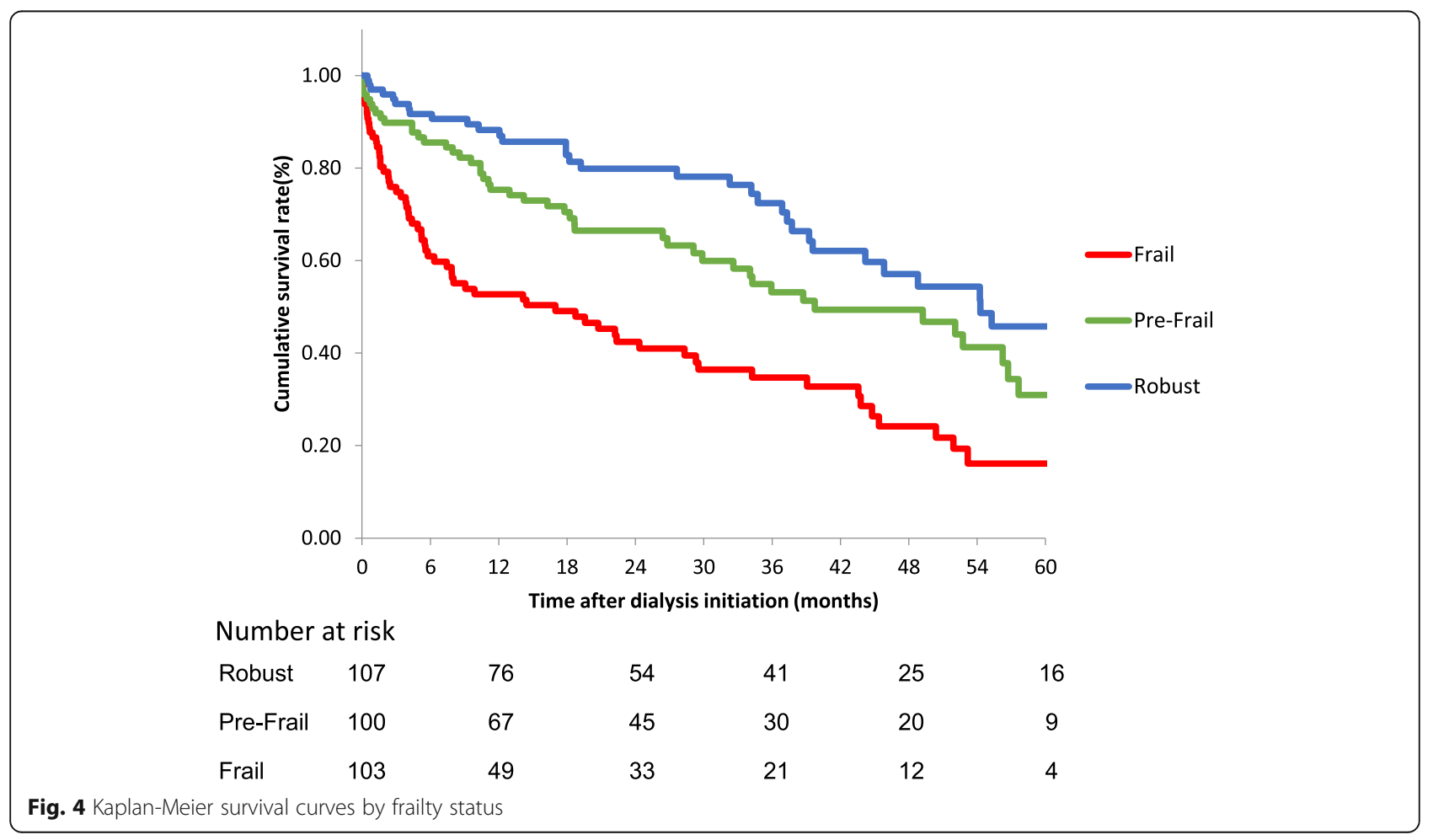




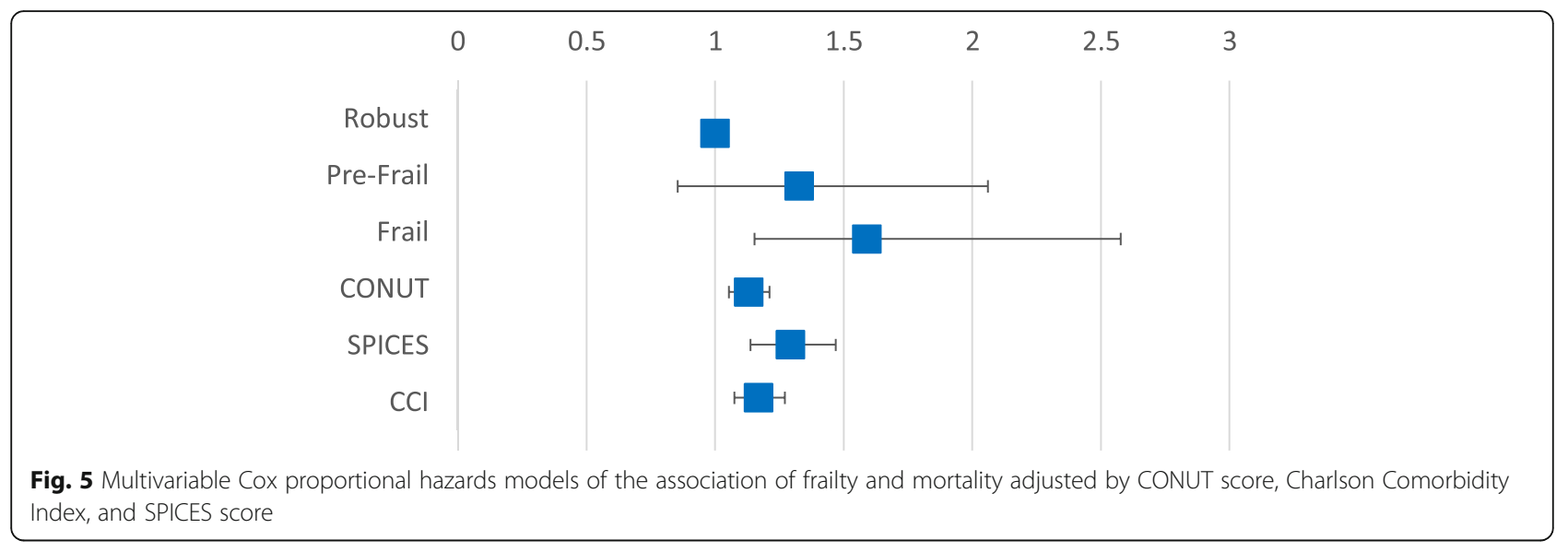

dementia in a patient with a frail status was 1.33 for all dementias (hazard ratio of 2.70 for vascular dementia and 1.28 for Alzheimer's-type dementia, respectively) [26]. Combined frailty and cognitive dysfunction tend to reduce the activities of daily living and physical function and increase mortality [27, 28].

Although no significant difference was observed in other comorbidities, the risk of frailty is considered to increase because of the accumulation of various comorbidities. In a previous study, the frequency of frail patients with acute coronary syndrome was $5.1-30 \%$, which was associated with increased death and readmission [29]. The frequency of heart failure among frail patients is $19-40 \%$, which is higher than that of the general population and is associated with increased death and readmission [30]. In Japan, diabetic nephropathy is the largest primary cause of dialysis (42.5\%) [14]. Therefore, in CKD stages $4-5$, the incidence of diabetes is high. Diabetes increases the risk of becoming frail, and frailty increases the incidence of diabetes [31, 32]. Frail patients with diabetes have a poor prognosis [33]. The prevalence of frailty in elderly patients with chronic obstructive pulmonary disease (COPD) is reported to be $10.2 \%$, and frailty is associated with physical dysfunction, and it is also a prognostic predictor. Frailty has the greatest effect on the prognosis of COPD patients [34, 35]. Early management of chronic comorbidity leads to the prevention of frailty and prevents deterioration of frailty progression because frailty and chronic comorbidity interact.

\section{Geriatric syndrome}

The simple-to-use screening strategy alerts the bedside nurse to be vigilant in the surveillance of patients and to initiate care team activities. SPICES is recommended by the Nurses Improving Care for Health System Elders (NICHE) as a valuable screening tool for identifying frailty risk among hospitalized older patients, and it is commonly used for this purpose.

The SPICES score was significantly higher in the frail group. Significant differences were observed in the following constituent factors: skin integrity, problem with eating, incontinence, confusion, and evidence of falls. Some factors can cause outcomes, as well as frailty, which can affect interactivity and accelerate the negative cascade. Major outcomes of frailty include falls/fractures, need for nursing care (decreased ability to perform routine activities of daily living, assistance with excretion), and death [36].

Exercise interventions for the frail are recommended to improve gait, muscle strength, physical motor function, and daily activities, and to prevent the progression of frailty. Exercise intervention for disability in daily activities should be implemented early in frail patients [37]. However, this study has some limitations. The SPICES score is considered competent, but the scale used to assess activities of daily living, sleep disorder, and confusion varies among reports. In the future, it will be necessary to establish unified evaluation criteria by repeating cases.

\section{Malnutrition}

Malnutrition and frailty are frequent among the older population [38]. An index of malnutrition includes Alb, BMI, total cholesterol, total lymphocyte count, and weight loss, among others [18, 39-41]. Compared with the other groups, Alb, BMI, and total lymphocyte count were significantly lower in the frail group.

The CONUT score is a screening tool to identify malnutrition by consideration of the protein reserves (Alb value), caloric depletion (total cholesterol), and immune defense (lymphocyte count) [18]. There have been many reports about the association between each component of CONUT score and outcomes. The drop in the lymphocyte count, which is caused by physical stress, malnutrition, and chronic inflammation, predicts the 1- 
Table 2 Cox proportional hazard models

\begin{tabular}{|c|c|c|c|c|c|c|c|c|}
\hline \multirow[t]{2}{*}{ Characteristic } & \multicolumn{4}{|c|}{ Univariate Cox proportional hazard models } & \multicolumn{4}{|c|}{ Multivariate Cox proportional hazard models } \\
\hline & $\beta$ coefficent & $P$-value & HR & $95 \% \mathrm{Cl}$ for $\mathrm{HR}$ & $\beta$ coefficent & $P$-value & HR & $95 \% \mathrm{Cl}$ for $\mathrm{HR}$ \\
\hline Age (year) & 0.0100 & 0.637 & 1.010 & $0.969-1.053$ & & & & \\
\hline Sex (Female) & -0.2700 & 0.122 & 0.763 & $0.542-1.075$ & & & & \\
\hline Body mass index (kg/m2) & 0.0160 & 0.706 & 1.016 & $0.935-1.104$ & & & & \\
\hline Cardio-Thoracic Ratio (\%) & 0.0320 & 0.008 & 1.032 & $1.008-1.056$ & & & & \\
\hline Serum Albumin (g/dl) & -0.4740 & 0.028 & 0.622 & $0.338-1.147$ & & & & \\
\hline Hemoglobin $(\mathrm{g} / \mathrm{dl})$ & 0.0180 & 0.780 & 1.018 & $0.900-1.151$ & & & & \\
\hline Blood Urea Nitrogen (mg/dl) & 0.0060 & 0.944 & 1.001 & $1.010-1.041$ & & & & \\
\hline Serum Creatinine (mg/dl) & 0.1220 & 0.656 & 1.130 & $0.680-1.877$ & & & & \\
\hline Serum Sodium (mEq/l) & 0.0150 & 0.435 & 1.015 & $0.978-1.053$ & & & & \\
\hline Serum Potassium (mEq/l) & 0.1320 & 0.104 & 1.141 & $0.973-1.339$ & & & & \\
\hline Serum Chloride (mEq/l) & -0.0360 & 0.028 & 0.965 & $0.934-0.996$ & & & & \\
\hline Corrected Serum Calcium (mg/dl) & -0.1670 & 0.099 & 0.846 & $0.694-1.032$ & & & & \\
\hline Serum Phosphorus (mg/dl) & 0.0570 & 0.414 & 1.059 & $0.923-1.214$ & & & & \\
\hline Brain Natriuretic Peptide (pg/ml) & 0.0002 & 0.009 & 1.000 & $1.000-1.000$ & & & & \\
\hline Total Cholesterol (mg/dl) & -0.0027 & 0.123 & 0.997 & $0.994-1.001$ & & & & \\
\hline Total Lymphocyte Count (1000/uL) & -0.5900 & 0.001 & 0.554 & $0.392-0.784$ & & & & \\
\hline C-reactive protein (mg/dl) & 0.6200 & 0.001 & 1.064 & $1.042-1.087$ & & & & \\
\hline CONUT score & 0.1806 & 0.000 & 1.198 & $1.119-1.283$ & 0.122 & 0.001 & 1.130 & $1.05-1.212$ \\
\hline SPICES Score & 0.2510 & 0.001 & 1.286 & $1.107-1.494$ & 0.230 & 0.002 & 1.293 & $1.138-1.469$ \\
\hline Charlson Comorbidity Index & 0.1290 & 0.012 & 1.137 & $1.029-1.257$ & 0.150 & 0.001 & 1.169 & $1.075-1.272$ \\
\hline \multicolumn{9}{|l|}{ Clinical Fraility Scale } \\
\hline Robust & & 1.395 & referance & & & 0.169 & referance & \\
\hline Pre-Frail & 0.4954 & 0.025 & 1.641 & $1.066-2.527$ & 0.283 & 0.209 & 1.327 & $0.85-2.061$ \\
\hline Frail & 1.1424 & 0.000 & 3.134 & $2.075-4.734$ & 0.464 & 0.040 & 1.591 & $1.15-2.577$ \\
\hline
\end{tabular}

year outcome in patients [42]. Alb is the most abundant protein in human serum. It has been used for decades as an indicator of malnutrition in patients in clinically stable conditions [43]. There is a strong relationship between Alb concentration and all-cause mortality in elderly subjects [44]. Conversely, a low Alb concentration in patients with CKD could be related to non-nutritional conditions, such as inflammation, acute or chronic comorbidities or infectious events, edema, fluid overload, and proteinuria. It remains debatable as to whether low levels of Alb in patients with CKD are a surrogate of inadequate protein intake, fluid overload, or other conditions related to protein-energy wasting, such as inflammation or comorbidity. However, there seems to be less disagreement regarding the consistent association of hypoalbuminemia with poor outcomes in CKD patients [45]. Compared with the other groups, CRP was significantly higher in the frail group. When assessing the Alb values, inflammatory status should be taken into account. CRP-adjusted
Alb was shown to be a better predictor of mortality among dialysis patients. Malnutrition or wasting was shown to be associated with a poor outcome independent of inflammation [46].

As this study is targeted at patients in the dialysis initiation stage, low Alb levels could be a result of malnutrition, but also fluid overload. Therefore, it was considered that all patients had low Alb levels $(<3.5 \mathrm{~g} / \mathrm{dL})$, even compared with a previous report that used Alb $3.8 \mathrm{~g} / \mathrm{dL}$ as a cutoff value for dialysis patients. CTR and BNP, which suggest fluid overload, also tended to be high. There is also a report that the state of congestive heart failure and edema with poor fluid control at the introduction of dialysis affects the prognosis of patients (HR, 1.867; 95\% CI, 1.467-2.376) [47].

Total cholesterol levels are a good reflection of dietary intake $[48,49]$. A systematic review and meta-analysis that investigated blood biomarkers associated with the risk of malnutrition in older adults found that total 


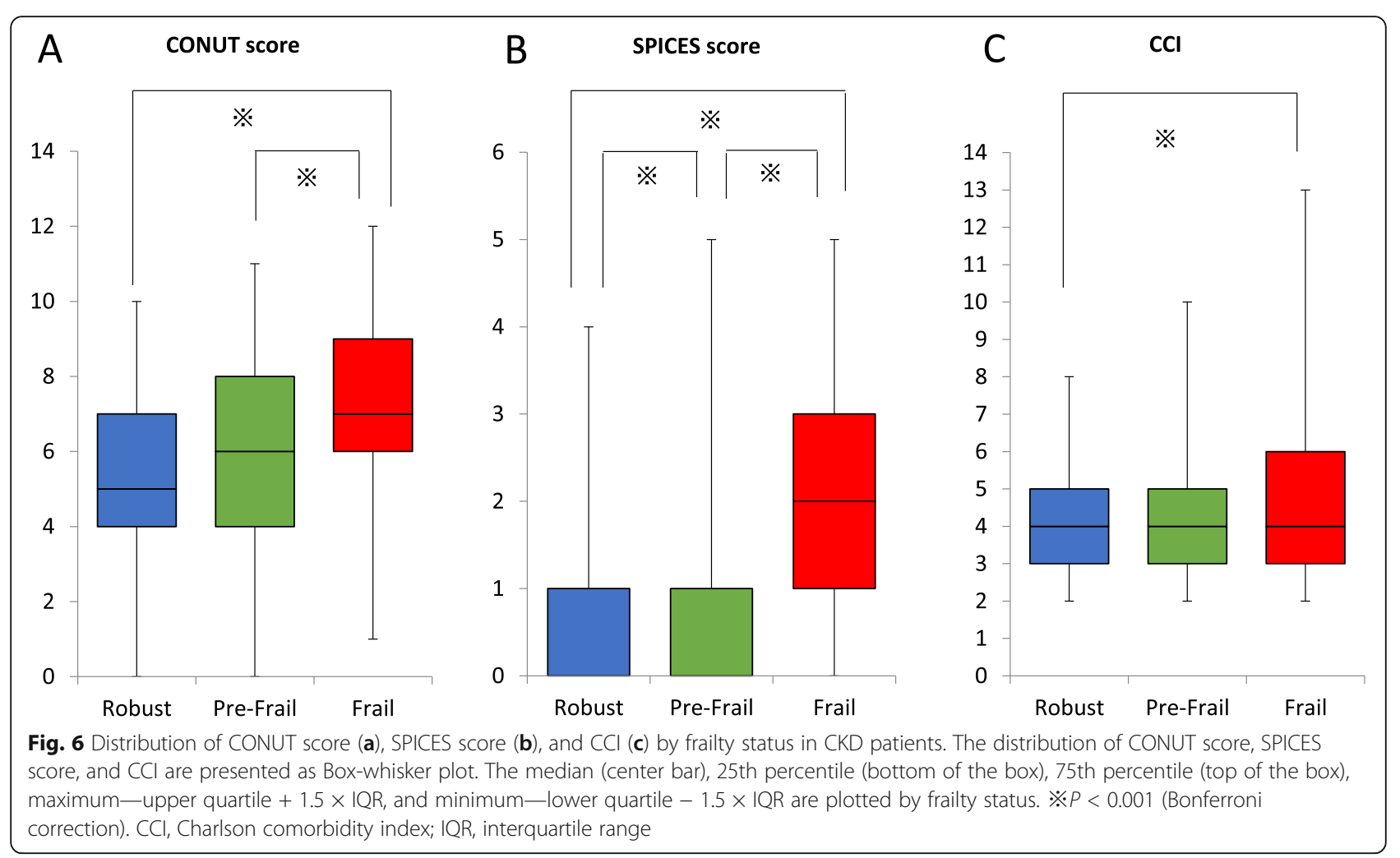

cholesterol was useful for the identification of malnutrition in older adults [50].

These underlying mechanisms were closely linked to not only the nutrition but also to the acute exacerbation of the comorbidity disease. Therefore, the CONUT score, an index of immunity status, protein reserve, and lipid metabolism, is hypothesized to have a significant impact on CKD and frail patients.

CFS and the three indicators highlighted in this study (CONUT score, SPICES score, and CCI) are associated with increased mortality. Especially for frail $(\mathrm{CFS} \geq 5$ ), each index showed significantly higher values. Also, there is a positive correlation between the three indicators. In other words, it seems reasonable to expect that if one indicator deteriorates, the other indicator will also deteriorate and become a negative spiral.

\section{Limitations}

This study had several limitations. Firstly, this study was performed using the database of a single center. Further generalizability of the present investigation will be required using the data of other cohorts. Second, only the baseline data were analyzed, and no changes in clinical indices, including CONUT score (Alb, total cholesterol, total lymphocyte count values), were considered. Substantial changes in the clinical parameters could occur afterward during the course. Thirdly, it is unclear if intervention for frailty at the time of dialysis induction would improve patient survival. This study does not assess the effect of interventions on frailty.

However, this study had several advantages. First, the follow-up period was also sufficient to investigate, and about $90 \%$ of patients could be followed up continuously from the time of introduction.

\section{Conclusion}

Frailty is caused by a variety of factors besides aging. This study showed the relationship between CFS and CONUT score, CCI, and SPICES score in consideration of prognosis. As far as we know, there are no reports evaluating

Table 3 Correlation coefficients for indices of CONUT score, SPICES score, CCI, and CFS

\begin{tabular}{lllll}
\hline & CONUT score & SPICES score & CCI & CFS \\
\hline CONUT score & 1 & $0.348(P<0.001)$ & $0.156(P=0.006)$ & $0.299(P<0.001)$ \\
SPICES score & $0.348(P<0.001)$ & 1 & $0.244(P<0.001)$ & $0.505(P<0.001)$ \\
CCI & $0.156(P=0.006)$ & $0.244(P<0.001)$ & 1 & $0.166(P=0.003)$ \\
CFS & $0.299(P<0.001)$ & $0.505(P<0.001)$ & $0.166(P=0.003)$ & 1 \\
\hline
\end{tabular}


relationships of these indices and prognosis in the same patients with CKD. The CFS allows frailty to be defined and graded using simple clinical descriptors available from routine clinical assessment. The prognosis after initiation of dialysis is poor if the patient is frail during the preservation period. Therefore, a multifaceted intervention is needed. In other words, chronic disease management, nutrition management, and coping with the cognitive and physical decline due to aging are necessary. However, it is unclear if intervention for frailty at the time of dialysis induction would improve patient survival. As a future study topic, it would be valuable to examine whether the prognosis is improved by the intervention for frailty, such as a multifaceted intervention

\section{Supplementary Information}

The online version contains supplementary material available at https:/doi. org/10.1186/s41100-020-00300-0.

\section{Additional file 1.}

\section{Abbreviations}

ADL: Activities of daily living; Alb: Serum albumin; BMI: Body mass index; BNP: Brain natriuretic peptide; CCl: Charlson Comorbidity Index; CFS: Clinical Frailty Scale; CHS: Cardiovascular Health Study; Cl: Confidence interval; CKD: Chronic kidney disease; CONUT: Controlling Nutritional Status; CRP: Creactive protein; CTR: Cardiothoracic ratio; eGFR: Estimated glomerular filtration rate; FI: Frailty index; FP: Frailty phenotype; IQR: Interquartile range; QOL: Quality of life

\section{Acknowledgements}

We are grateful to the members of the Dialysis and Nephrology Center, Sanshikai TOHO Hospital, for their important contributions to the treatment of patients.

\section{Authors' contributions}

All authors collaborated in the data collection and analysis. As a dialysis and nephrology center administrator, KU contributed to the writing of the manuscript. All authors have read and approved the final manuscript.

\section{Funding}

The funding institution for the research design, data collection, analysis, interpretation, and manuscript writing was the Sanshikai Toho Hospital.

\section{Availability of data and materials \\ The datasets analyzed during the current study are available from the corresponding author upon reasonable request.}

\section{Ethics approval and consent to participate}

The study protocol and informed consent document were reviewed and approved by the ethical standards of the Sanshikai Toho Hospital Ethics Committee for Clinical Research, Gunma, Japan (IRB 19-0021). The procedures were conducted in accordance with the 1964 Helsinki declaration and its later amendments or comparable ethical standards.

\section{Consent for publication}

All patients have signed a comprehensive agreement to use the collected samples that accompany their treatment for future medical research. The contents of the research are thoroughly deliberated by the Ethics Committee, and the data were used within the scope approved. If the patient refused or withdrew consent, there will be no penalty for medical treatment. The samples were anonymized to ensure that individuals could not be identified, and the utmost care was taken to protect personal privacy.

\section{Competing interests}

The authors declare that they have no competing interests.

Received: 25 May 2020 Accepted: 28 October 2020

Published online: 07 December 2020

\section{References}

1. Clegg A, Young J, lliffe S, et al. Frailty in elderly people. Lancet. 2013;381:752-62.

2. Kojima G, lliffe $S$, Taniguchi $Y$, et al. Prevalence of frailty in Japan: a systematic review and meta-analysis. J Epidemiol. 2017;27:347-53.

3. Fried LP, Tangen CM, Walston J, et al. Frailty in older adults: evidence for a phenotype. J Gerontol A Biol Sci Med Sci. 2001;56:M146-56.

4. Mitnitski AB, Mogilner AJ, Rockwood K. Accumulation of deficits as a proxy measure of aging. Sci World J. 2001;1:321027.

5. Rockwood K, Song X, MacKnight C, et al. A global clinical measure of fitness and frailty in elderly people. Can Med Assoc J. 2005;173:489-95.

6. Basic D, Shanley C. Frailty in an older inpatient population: using the clinical frailty scale to predict patient outcomes. J Aging Health. 2015;27:670-85.

7. Rockwood K, Abeysundera MJ, Mitnitski A. How should we grade frailty in nursing home patients? J Am Med Dir Assoc. 2007:8:595-603.

8. Dent E, Lien C, Lim WS, et al. The Asia-Pacific Clinical Practice Guidelines for the management of frailty. J Am Med Dir Assoc. 2017;18:564-75.

9. Nixon AC, Bampouras TM, Pendleton N, et al. Diagnostic accuracy of frailty screening methods in advanced chronic kidney disease. Nephron. 2019;141:147-55.

10. Lee SJ, Son H, Shin SK. Influence of frailty on health-related quality of life in pre-dialysis patients with chronic kidney disease in Korea: a cross-sectional study. Health Qual Life Outcomes. 2015;13:70.

11. Mansur HN, Colugnati FA, Grincenkov FR, et al. Frailty and quality of life: a cross-sectional study of Brazilian patients with pre-dialysis chronic kidney disease. Health Qual Life Outcomes. 2014;12:27-33.

12. Roshanravan B, Khatri M, Robinson-Cohen C, et al. A prospective study of frailty in nephrology-referred patients with CKD. Am J Kidney Dis. 2012;60: 912-21.

13. Alfaadhel TA, Soroka SD, Kiberd BA, et al. Frailty and mortality in dialysis: evaluation of a clinical frailty scale. Clin J Am Soc Nephrol. 2015;10:832-40.

14. Nitta K, Masakane S, Hanafusa N, et al. 2017 Annual dialysis data report, JSDT Renal Data Registry. J Jpn Soc Dialysis Ther. 2018:51:699-766.

15. Charlson ME, Pompei P, Ales KL, et al. A new method of classifying prognostic comorbidity in longitudinal studies: development and validation. J Chronic Dis. 1987;40(5):373-83. https:/doi.org/10.1016/0021-9681(87)90171-8.

16. Granger CV, Hamilton BB, Linacre JM, et al. Performance profiles of the functional independence measure. Am J Phys Med Rehabil. 1993;72:84-9.

17. Aronow HU, Borenstein J, Haus F, et al. Validating SPICES as a screening tool for frailty risks among hospitalized older adults. Nurs Res Pract. 2014:2014: 846759

18. Ignacio de Ulibarri J, Gonzalez-Madrono A, de Villar NG, et al. CONUT: a tool for controlling nutritional status. First validation in a hospital population. Nutr Hosp. 2005:20:38-45.

19. Japanese Society of Nephrology. Essential points from evidence-based clinical practice guidelines for chronic kidney disease 2018. Clin Exp Nephrol. 2019;23:1-15.

20. Walker SR, Gill K, Macdonald K, et al. Association of frailty and physical function in patients with non-dialysis CKD: a systematic review. BMC Nephrol. 2013;14:228.

21. Reese PP, Cappola AR, Shults J, et al. Physical performance and frailty in chronic kidney disease. Am J Nephrol. 2013;38:307-15.

22. Gordon Emily $H_{1}$ Hubbard Ruth $\mathrm{E}_{\text {, et }}$ al. Differences in frailty in older men and women. Med J Aust. 2020;212(4). https://doi.org/10.5694/mja2.50466.

23. Yazawa M, Kido R, Ohira S, Hasegawa T, Hanafusa N, Iseki K, et al. Early mortality was highly and strongly associated with functional status in incident Japanese hemodialysis patients: a cohort study of the large National Dialysis Registry. PLoS One. 11(6):e0156951 doi: 10.1371.

24. Tamura MK, Covinsky KE, Glenn M, Chertow, et al. Functional status of elderly adults before and after initiation of dialysis. N Engl J Med. 2009; 361(16):1539-47. https://doi.org/10.1056/NEJMoa0904655

25. Bilotta C, Bergamaschini L, Nicolini P, et al. Frailty syndrome diagnosed according to the Study of Osteoporotic Fractures criteria and mortality in older outpatients suffering from Alzheimer's disease: a one-year prospective cohort study. Aging Ment Health. 2012;16:273-80.

26. Kojima $G$, Taniguchi $Y$, lliffe $S$, et al. Frailty as a predictor of Alzheimer disease, vascular dementia, and all dementia among community-dwelling 
older people: a systematic review and meta-analysis. J Am Med Dir Assoc. 2016;17:881-8.

27. Solfrizzi V, Scafato E, Frisardi V, et al. Frailty syndrome and all-cause mortality in demented patients: the Italian Longitudinal Study on Aging. Age. 2012; 34:507-17.

28. Shimada $\mathrm{H}$, Makizako $\mathrm{H}$, Lee $\mathrm{S}$, et al. Impact of cognitive frailty on daily activities in older persons. J Nutr Health Aging. 2016;20:729-35.

29. Sanchis J, Bonanad C, Ruiz V, et al. Frailty and other geriatric conditions for risk stratification of older patients with acute coronary syndrome. Am Heart J. 2014;168:784-91.

30. McNallan SM, Singh M, Chamberlain AM, et al. Frailty and healthcare utilization among patients with heart failure in the community. JACC Heart Failure. 2013;1:135-41.

31. Gracía-Esquinas E, Graciani A, Guallar-Castillón P, et al. Diabetes and risk of frailty and its potential mechanisms: a prospective cohort study of older adults. J Am Med Dir Assoc. 2015;16:748-54

32. Veronese N, Stubbs B, Fontana L, et al. Frailty is associated with an increased risk of incident type 2 diabetes in the elderly. J Am Med Dir Assoc. 2016;17:902-7.

33. Castro-Rodríguez M, Carnicero JA, Garcia-Gracia FJ, et al. Frailty as a major factor in the increased risk of death and disability in older people with diabetes. J Am Med Dir Assoc. 2016;17:949-55.

34. Lahousse L, Ziere G, Verlinden VJA, et al. Risk of frailty in elderly with COPD: a population-based study. J Gerontol A Biol Sci Med Sci. 2016; 71:689-95.

35. Galizia G, Cacciatore F, Testa G, et al. Role of clinical frailty on long-term mortality of elderly subjects with and without chronic obstructive pulmonary disease. Aging Clin Exp Res. 2011;23:118-25.

36. Vermeiren S, Vella-Azzopardi R, Beckwée D, et al. Frailty and the prediction of negative health outcomes: a meta-analysis. J Am Med Dir Assoc. 2016;17: 1163.e1-17.

37. Bibas L, Levi M, Bendayan $M$, et al. Therapeutic interventions for frail elderly patients: part 1. Published randomized trials. Prog Cardiovasc Dis. 2014;57: $134-43$

38. Boulos C, Salameh P, Barberger-Gateau P. Malnutrition and frailty in community dwelling older adults living in a rural setting. Clin Nutr. 2016;35:138-43.

39. Hubbard RE, Lang IA, Llewellyn DJ, et al. Frailty, body mass index, and abdominal obesity in older people. J Gerontol A Biol Sci Med Sci. 2010;65A:377-81.

40. Kobayashi S, Asakura K, Suga $\mathrm{H}$, et al. High protein intake is associated with low prevalence of frailty among old Japanese women: a multicenter crosssectional study. Nutr J. 2013;12:164.

41. Vânia Aparecida Leandro-Merhi, Vitória Negri Bráz , José Luis Braga de Aquino et al. Is total lymphocyte count related to nutritional markers in hospitalized older adults? Arq Gastroenterol2017;54(1):79-82.doi:https://doi. org/10.1590/50004-2803.2017v54n1-16.

42. Bosma-den Boer MM, van Wetten M-L, Pruimboom L, et al. Chronic inflammatory diseases are stimulated by current lifestyle: how diet, stress levels and medication prevent our body from recovering. Nutr Metab. 2012; 9:32 http://www.nutritionandmetabolism.com/content/9/1/32.

43. Cabrerizo S, Cuadras D, Gomez-Busto F, et al. Serum albumin and health in older people: review and meta analysis. Maturitas. 2015;81:17-27.

44. Corti M-C, Guralnik JM, Salive ME, et al. Serum albumin level and physical disability as predictors of mortality in older persons. JAMA. 1994;272: 1036-42.

45. Carrero JJ, Thomas F, Nagy K, et al. Global prevalence of protein-energy wasting in kidney disease: a meta-analysis of contemporary observational studies from the international society of renal nutrition and metabolism. J Ren Nutr. 2018;28:380-92.

46. Hanafusa N, Nitta K, Tsuchiya K, et al. Serum albumin level adjusted with Creactive protein predicts hemodialysis patient survival. Renal Replacement Therapy. 2017:3(Article number: 9).

47. Yamagata K, Nakai S, Committee of Renal Data Registry of the Japanese Society for Dialysis Therapy, et al. Late dialysis start did not affect long-term outcome in Japanese dialysis patients: long-term prognosis from Japanese Society for [Corrected] Dialysis Therapy Registry. Ther Apher Dial. 2012;16(2): 111-20. https://doi.org/10.1111/j.1744-9987.2011.01052.x.

48. Horwich TB, Hernandez AF, Dai D, Yancy, et al. Cholesterol levels and inhospital mortality in patients with acute decompensated heart failure. Am Heart J. 2008;156:1170-6. https://doi.org/10.1016/..ahj.2008.07.004.
49. Mohammad MH, Abdullah PJH, Jones PKE. Nutrigenetics of cholesterol metabolism: observational and dietary intervention studies in the postgenomic era. Nutr Rev. 2015;73:523-43. https:/doi.org/10.1093/nutrit/nuv016.

50. Zhang Z, Pereira S, Luo M, Matheson E, et al. Evaluation of blood biomarkers associated with risk of malnutrition in older adults: a systematic review and meta-analysis. Nutrients. 2017;9(8):829.

\section{Publisher's Note}

Springer Nature remains neutral with regard to jurisdictional claims in published maps and institutional affiliations.

\section{Ready to submit your research? Choose BMC and benefit from:}

- fast, convenient online submission

- thorough peer review by experienced researchers in your field

- rapid publication on acceptance

- support for research data, including large and complex data types

- gold Open Access which fosters wider collaboration and increased citations

- maximum visibility for your research: over $100 \mathrm{M}$ website views per year

At BMC, research is always in progress.

Learn more biomedcentral.com/submissions 